\title{
Encountering a Very Rare Pancreatic Tumor-Solid Pseudopapillary Tumor of Pancreas
}

\section{Urmila Basu ${ }^{1}$, Subham Bera ${ }^{1}$, Devmalya Banerjee $^{2}$, Shubhayu Banerjee $^{3}$ and Gaurav Kumar ${ }^{4 *}$}

${ }^{1}$ DNB Resident, Department of General Surgery, Rabindranath Tagore International Institute of Cardiac Sciences, Kolkata, West Bengal, India

${ }^{2}$ Consultant Oncopathologist, Narayana Superspeciality Hospital, Kolkata, West Bengal, India

${ }^{3}$ Consultant Laparoscopic and Gastrointestinal Surgeon, Rabindranath Tagore International Institute of Cardiac Sciences, Kolkata, West Bengal, India

${ }^{4}$ Consultant General Surgeon, Narayana Superspeciality Hospital, Kolkata, West Bengal, India

*Corresponding Author: Gaurav Kumar, Consultant General Surgeon, Narayana Superspeciality Hospital, Kolkata, West Bengal, India.
Received: February 05, 2020

Published: March 31, 2020

(C) All rights are reserved by Gaurav Kumar., et al.

\begin{abstract}
Solid pseudopapillary tumor of pancreas is a very rare tumor of low malignant potential accounting for only $2-3 \%$ of all pancreatic neoplasms. It affects females of African American ethnicity. The tumor biology is unique in itself. It is seen that vascular invasion, degree of nuclear atypia, mitotic count and prominence of necrobiotic cell nests (cells with pycnotic nuclei and eosinophilic cytoplasm) are associated with malignancy. However, neoplasms in which above mentioned criteria of malignancy are not detected may also give rise to metastases. Hence benign appearing SPNs must be classified as lesions of uncertain malignant potential and must be operated on. Here we present a 27-year-old lady with $3 \mathrm{~cm}$ space occupying lesion in the head of the pancreas. Endoscopic ultrasonography guided biopsy revealed Solid Pseudopapillary Tumor of pancreas. Pancreaticoduodenectomy was carried out without significant complications and she was discharged on post-operative day 7 . As this is a very rare tumor one encounters, we thought of reporting it.

Keywords: Pancreas; Solid Pseudopapillary Tumor of pancreas; Histology; Immunohistochemistry; Treatment; ancreaticoduodenectomy
\end{abstract}

\section{Abbreviations}

SPN: Solid Pseudopapillary Neoplasm of Pancreas; MPD: Main Pancreatic Duct; GDA: Gastro Duodenal Artery; EUS: Endoscopic Ultrasonography; SOL: Space Occupying Lesion; POD: Post-Operative Day

\section{Introduction}

The first report of SPN is credited by Lichtenstein [1] who resected a tumor of the pancreatic tail which from the description could have been a pseudopapillary tumor. Solid pseudopapillary neoplasm (SPN) of pancreas were initially described by Frantz in 1959 [2] and the electron microscopic features by Hamoudi., et al. in 1970 [3]. In 1996 it was reclassified as solid pseudopapillary tumor with borderline malignant potential by World Health Organization (WHO) [4].

The origin of solid pseudopapillary neoplasm of the pancreas is unknown. It is a rare epithelial neoplasm. It contributes to $2 \%$ - 3\% of pancreatic tumors in adults but unlike other pancreatic neoplasms, it is more prevalent in females. SPN has a low rate of malignancy (10 - 15\%) [5] and seldom metastasizes. Even in the event of disseminated cases, surgical treatment is a viable option with a favorable outcome. Due to phenomenal advances in CT and MRI there has been an apparent increase in detection of SPN during the last 15 years, as most of the tumors are asymptomatic and detected incidentally [6].

Here we present a 27-year-old lady with SPN at the head of pancreas who underwent Pancreaticoduodenectomy in our institute.

\section{Case Report}

A 27-year-old lady presented to us with intermittent upper abdominal pain, anorexia and weight loss. On further questioning she did mention that her symptoms were noticeable for the past 3 months. She denied any episode of fever, jaundice, nausea, vomiting, hematemesis or malena. There was no past surgical or medical history. General and systemic examinations did not reveal any stigmata attributable to the presenting disease. Abdominal examination revealed mild tenderness in the epigastric region with no evidence of any palpable lump. 
Her routine blood investigation including Liver Function Test, Amylase and Lipase were within normal limit. CA 19-9 was 12.6 $\mathrm{U} / \mathrm{ml}$.

Ultrasonography confirmed A $26 \mathrm{~mm}$ X $25 \mathrm{~mm}$ hypoechoic SOL in the head of the pancreas which could not be separated from the pancreas and $2 \mathrm{~mm}$ polyp on anterior wall of GB. Triple Phase CT (Figure 1) confirmed A hypodense exophytic space occupying lesion (32 X 34 X $29 \mathrm{~mm}$ ) in size arising from the head of the pancreas with tiny cystic non enhancing areas inside. There was partial encasement of adjacent Gastro- duodenal artery and loss of fat planes with posterior antral wall.
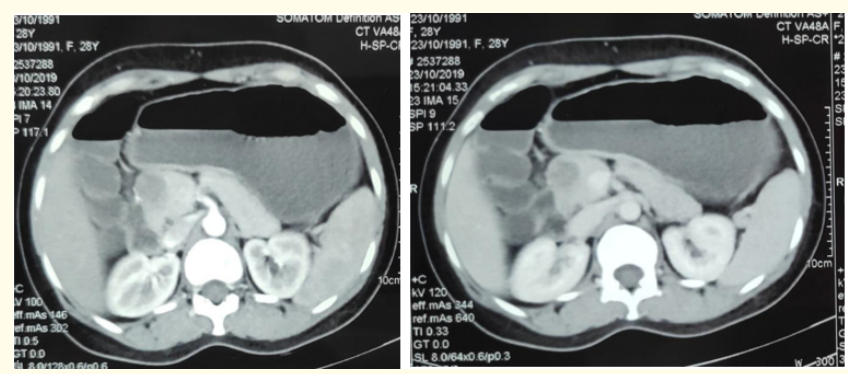

Figure 1: CT scan images of mass lesion arising from the head of pancreas.

Endoscopic ultrasonography confirmed Hypoechoid SOL (22 $\mathrm{X} 24 \mathrm{~mm}$ ) in the pancreatic head with a distinct tissue planes between GDA and the SOL unlike the CT scan findings. MPD was normal in caliber ( $2 \mathrm{~mm}$ in the head). EUS guided FNAC was done whose cytomorphology was compatible with Solid Pseudopapillary tumor of pancreas.

She underwent a Whipples procedure on 23/11/2019. Technically it was a challenging surgery because of dense adhesions encountered during the retropancreatic dissection. Generally, in our Institute we perform a pancreaticojejunostomy, but in this case as the MPD was undilated, we had no option but to do a pancreaticogastrostomy (Figure 2). Her post-operative recovery was uneventful and she was discharged on Day 7.

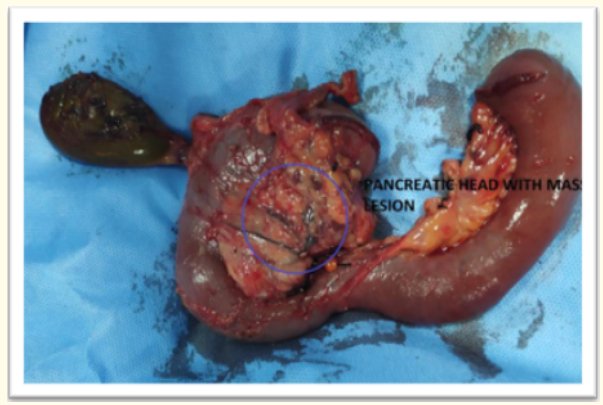

Figure 2: Post-operative specimen of pancreaticoduodenectomy showing a mass lesion arising from the head of pancreas.
Histopathological examination of specimen confirmed a gray white circumscribed tumor measuring $3.0 \times 3.0 \times 3.5 \mathrm{~cm}$ with all tumor free margins with few reactive Lymph nodes.

Tumor cells with dis-cohesive growth pattern arranged in a pseudo papillary architecture. Mitosis, necrosis, and atypical nuclei were absent (Figure 3). On Immunohistochemistry tumor cells are diffusely positive for cytoplasmic and nuclear staining of beta catenin (Figure 4). Whereas negative for cytokeratin and chromogranin (Figure 5). A less than 1\% Ki67 proliferative index was established (Figure 6).
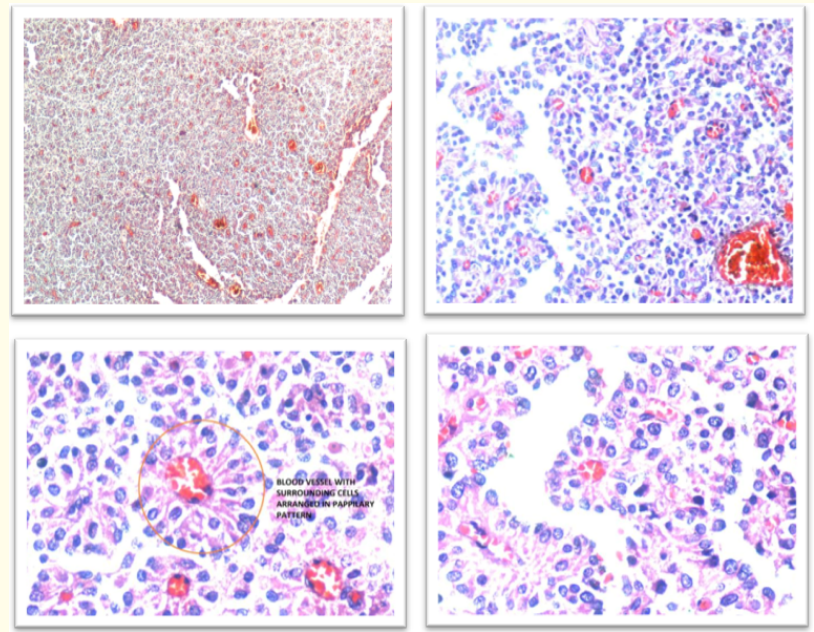

Figure 3: Hematoxylin eosin images of SPN.
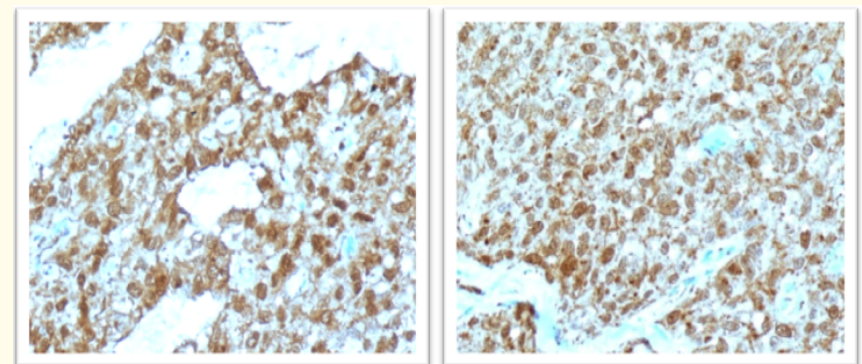

Figure 4: Immunohistochemistry for beta catenin.

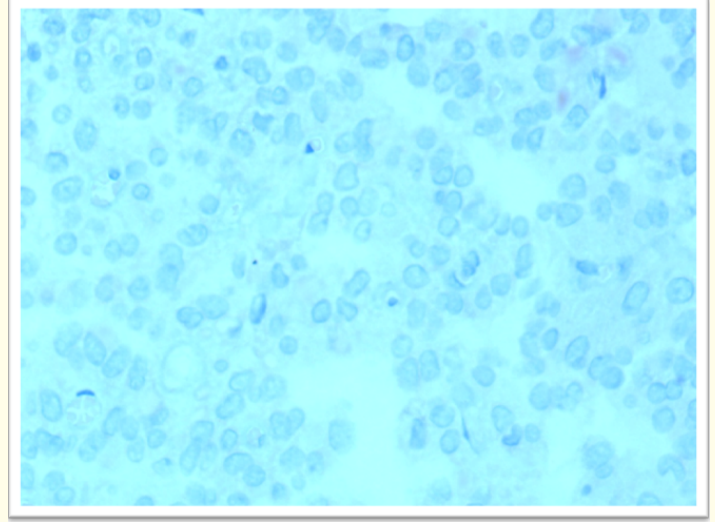

Figure 5: Immunohistochemistry for synaptophysin. 


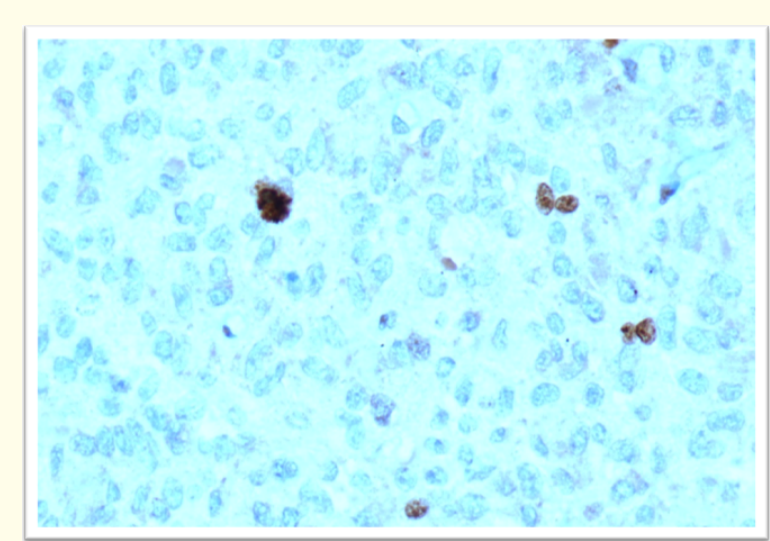

Figure 6: Immunohistochemistry for Ki67.

\section{Discussion}

The SPN of the pancreas is a special entity as it has no resemblance to other pancreatic tumors. It has been postulated that the tumor cells might have originated from a source other than the pancreas [7]. As we are aware that SPN is more prevalent in females, it is possible that these tumors may develop from displaced cells from the ovarian genital ridge, which is close to the primordial pancreas. The other hypothesis is that under the influence of female hormones this tumor originated from pluripotent embryonic cells in the pancreas. A study was conducted by Carsten., et al. on 1661 who underwent pancreatic resection from January 2001 to September 2018. Out of 1661, 15 patients were recorded to have SPN of which 12 were females [8]. An Indian study by TB Patil., et al. from 1992 - 2002 on 14 patients where all 14 were females [9]. Another study by He Song., et al. on 53 patients out of which 46 were females [10]. All these studies clearly reveal the female preponderance of the tumor. As expected our patient was also a female.

Pain or non-specific abdominal symptoms are the most common presenting complaints, but tumor may also be asymptomatic and, in such a case attains a considerable size before diagnosis. A few patients may present with nausea and vomiting attributable to alimentary tract compression by the tumor. However, there is no correlation between tumor size and symptoms. Even if the tumor is present in the head of the pancreas it unlikely to cause obstructive jaundice or main pancreatic duct obstruction. Our patient had a 3 $\mathrm{X} 3 \mathrm{~cm}$ tumor in the head of the pancreas and presented with pain abdomen and loss of appetite, she had no features of obstructive jaundice.

SPN in usually an encapsulated tumor with solid and cystic components, occasionally with rim-like calcification in the capsule as well as intra-parenchymatous calcifications on imaging studies (Ultrasonography, CT scan and MRI) [11,12]. Margins are well defined without pancreatic duct dilatation. Ultrasonography confirms echogenic and hypoechogenic components, CT scan depicts areas of varying density. On MRI, there is high-signal intensity on
$\mathrm{T} 1$ and low signal intensity on $\mathrm{T} 2$ series representing hemorrhagic areas in the lesions [12]. The tumor is usually found to be avascular or hypovascular on angiography. These radiological features are also seen in other pancreatic tumors especially cystic neuroendocrine tumors and pancreatoblastoma. The tumor cells may have high as well as low metabolism therefore showing variable activity in 18F-FDG-PET [13].

The pseudopapillary structure of SPN comprises of cells arranged in several layers around fibrovascular stalks. The histological characteristic of a SPN are pseudopapillary architecture, hyaline globules, cholesterol clefts, foamy macrophages, and nuclear grooving with the absence of neuroendocrine (salt-and-pepper) chromatin $[14,15]$. On electron microscopy we can see electrondense granules that may contain $\alpha-1$ antitrypsin and non-desmosome- like junction [14]. Both nucleus and cytoplasm of SPN is positive for $\beta$-catenin and there loss of E-cadherin from the cytoplasmic membrane revealed on immunohistochemistry [16,17]. Progesterone receptor, $\alpha-1$ antitrypsin receptor, and CD10 are some common positive markers [18]. Neuroendocrine markers such as synaptophysin, neuron-specific enolase and chromogranin are often expressed in SPNs. Electron microscopy may be useful if sufficient information cannot be attained with histology and immunohistochemistry.

Pancreatoblastoma, acinic cell tumor, and neuroendocrine tumor bear several radiographical and immunohistochemical similarities to SPN $[19,20]$. Pancreatoblastoma expresses nuclear and cytoplasmic $\beta$-catenin, but their histologic appearance differs from SPN due to the presence of squamoid corpuscles and dense bands of fibrous stroma [21]. Unlike SPN, Pancreatoblastoma is more prevalent in males and most commonly affects the pediatric age group. Acinic cell tumors simulate pancreatoblastoma in histologic appearance but are devoid of squamoid corpuscles. They rarely affect children. SPN often express synaptophysin, chromogranin, and CD56 neuroendocrine tumor markers. Functioning Neuroendocrine tumors (islet-cell tumors) secrete hormones. But non-functioning neuroendocrine tumors and SPNs cannot be easily distinguished.

The only definite sign of malignancy in SPN is metastases making differentiation between SPN with malignant and benign potential difficult [22]. WHO classifies SPN as solid-pseudopapillary carcinoma, in case of nerve sheath invasion or lymph node and liver metastases. The relative indicators of malignancy are tumor size larger than $5 \mathrm{~cm}$, capsular invasion and peripancreatic spread. Nishihara., et al. compared the histological features of 3 metastasizing and 19 non metastasizing SPN and found venous invasion, degree of clear atypia mitotic count and prominence of necrobiotic cell nests (cells with pycnotic nuclei and eosinophilic cytoplasm) were associated with malignancy. However, neoplasms in which above mentioned criteria of malignancy are not detected may also 
give rise to metastases. Consequently, benign appearing SPNs must be classified as lesions of uncertain malignant potential.

Pathology and immunohistochemistry are the main tools in the diagnosis of SPN. On immunohistochemistry Vinmentin, AACT, AAT, NSE, Synaptophysin, and PR are frequently positive. Malignant potential and outcome can be predicted by Ki-67 index [2325], however some studies have disregarded its association with malignancy [26]. Malignant SPN with low proliferative index of Ki-67 ( $\leq 5 \%)$ are slow growing and have more favorable outcome.

Radical surgery with R0 resection margin is the only curative treatment [27-29] localized growth of tumor makes this is possible in most cases. Adjuvant oncologic therapy does not improve survival. Tumor size, location and intraoperative frozen section examination dictates the extent of surgery. Since our patient had a pancreatic head tumor she underwent a Whipples procedure. Tumors with local infiltration or metastatic disease are also cured with radical resection including all metastatic tissue. The 2-year and 5-year survival rates are $97 \%$ and $95 \%$ respectively as reported in a study conducted by Papavramidis and Papavramidis on 467 patients [30]. According to a study conducted by Law., et al. on 1952 cases $4.4 \%$ patients suffered recurrence; while $1.5 \%$ patients died of SPN.

Various oncological protocols comprising of 5-FU, S-1, gemcitabine, sunitinib, and transarterial embolization of liver metastases (TACE) have been used for primary metastatic disease as well as recurrence [31,32]. Despite all of these treatment modalities, aggressive surgical resection remains the gold standard for primary metastatic and recurrent SPN.

\section{Conclusion}

To summarize, all the available literature suggests pancreatic SPN to be a rare neoplasm with a malignant potential having a favorable prognosis. Even in a backdrop of metastatic disease radical surgery is associated with improved long-term survival. As previously discussed recurrence should be treated surgically since it is well documented that medical oncological treatment has limited benefits.

\section{Bibliography}

1. Lichtenstein L. "Papillary cystadenocarcinoma of pancreas: case report, with notes on classification of malignant cystic tumors of pancreas". The American Journal of Cancer 21.3 (1934): 542-53.

2. Frantz VK. "Tumors of the pancreas". Armed Forces Institute of Pathology (1959).

3. Hamoudi AB., et al. "Papillary epithelial neoplasm of pancreas in a child. Report of a case with electron microscopy". Cancer 26.5 (1970): 1126-1134.
4. Klöppel G., et al. "Histological typing of tumours of the exocrine pancreas". Springer Science and Business Media 49.9 (1996): 780.

5. Mulkeen AL., et al. "Less common neoplasms of the pancreas". World Journal of Gastroenterology 12.20 (2006): 3180-3185.

6. Law JK., et al. "A systemic review of solid pseudopapillary neoplasm: are these rare lesions". Pancreas 43.3 (2014): 331-337.

7. Kosmahl M., et al. "Solid-pseudopapillary tumor of the pancreas: its origin revisited". Virchows Archiv 436.5 (2000): 473-480.

8. Hansen CP., et al. "Solid pseudopapillary neoplasm of the pancreas: Clinical-pathological features and management, a single-center experience". Rare Tumors (2019).

9. Patil TB., et al. "Solid pseudopapillary neoplasm of the pancreas: a single institution experience of 14 cases". HPB 18.2 (2006): 148-150

10. Song H., et al. "Solid pseudopapillary neoplasm of the pancreas: Clinicopathologic feature, risk factors of malignancy, and survival analysis of 53 cases from a single center". Bio Med Research International (2017): 5465261.

11. Park MJ., et al. "Multidetector CT imaging features of solid pseudopapillary neoplasm of the pancreas in male patients: distinctive imaging features with female patients". British Institute of Radiology 87.1035 (2014): 20130513.

12. Ventriglia A., et al. "MRI features of solid pseudopapillary neoplasm of the pancreas". Abdominal Imaging 39.6 (2014): 12131220.

13. Park M., et al. "Metabolic characteristics of solid pseudopapillary neoplasms of the pancreas: their relationships with high intensity 18F-FDG PET images". Oncotarget 9.15 (2018): 12009-12019.

14. Klimstra DS., et al. "Solid pseudo- papillary tumor of the pancreas: a typically cystic carcinoma of low malignant potential". Seminars in Diagnostic Pathology 17.1 (2000): 66-80.

15. Meriden Z., et al. "Hyaline globules in neuro-endocrine and solid - pseudopapillary neoplasms of the pancreas: a clue to the diagnosis". The American Journal of Surgical Pathology 35.7 (2011): 981-988.

16. Kim MS., et al. "Accumulation of beta catenin protein, mutations in exon- 3 of the beta catenin gene and a loss of heterozygosity of 5q22 in solid pseudopapillary tumor of pancreas". Journal of Surgical Oncology 94.5 (2006): 418-490.

17. Terris B and Cavard C. "Diagnosis and molecular aspects of solid-pseudopapillary neoplasms of the pancreas". Seminars in Diagnostic Pathology 31.6 (2014): 484-490. 
18. Ohara Y., et al. "Pancreatic neuroendocrine tumor and solidpseudopapillary neoplasm: key immuno- histochemical profiles for differential diagnosis(Observational study)". World Journal of Gastroenterology 22.38 (2016): 8596-8604.

19. Chung EM., et al. "Pancreatic tumors in children: radiologicpathologic correlation". Radio Graphics 26.4 (2006): 12111238.

20. Hackeng WM., et al. "Surgical and molecular pathology of pancreatic neoplasms”. Diagnostic Pathology 11.1 (2016): 47.

21. Klimstra DS., et al. "Pancreatoblastoma. A clinicopathologic study and review of the literature". The American Journal of Surgical Pathology 19.12 (1995): 1371-1389.

22. Kang CM., et al. "Predicting recurrence of pancreatic solid pseudopapillary tumors after surgical resection: a multicenter analysis in Korea". Annals of Surgery 260.2 (2014): 348-355.

23. Serra S and Chetty R. "Revision 2: an immunohistochemical approach and evaluation of solid pseudopapillary tumour of the pancreas". Journal of Clinical Pathology 61.11 (2008): 1153-1159.

24. Yang F., et al. "Solid pseudopapillary tumor of the pancreas: a case series of 26 consecutive patients". The American Journal of Surgery 198.2 (2009): 210-215.

25. Watanabe Y., et al. "A case of aggressive solid pseudopapillary neoplasm: Comparison of clinical and pathologic features with non-aggressive cases". Pathology International 67.4 (2017): 202-207.

26. I Hosokawa., et al. "Preoperative diagnosis and surgical management for solid pseudopapillary neoplasm ofd the pancreas". Journal of Hepato-Biliary-Pancreatic Sciences 21.8 (2014): 573-578.

27. El Nakeeb A., et al. "Solid pseudopapillary tumour of the pancreas: Incidence, prognosis and outcome of surgery (single center experience)". International Journal of Surgery 11.6 (2013): 447-457.

28. Lubezky N., et al. "Solid pseudopapillary neoplasm of the pancreas: management and long-term outcome". European Journal of Surgical Oncology (EJSO) 43.6 (2017): 1056-1060.

29. Reddy S., et al. "Surgical management of solid-pseudopapillary neoplasms of the pancreas (Franz or Hamoudi tumors): a large single-institutional series". Journal of the American College of Surgeons 208.5 (2009): 950-957.

30. Papavramidis T and Papavramidis S. "Solid pseudopapillary tumors of the pancreas: review of 718 patients reported in English literature". Journal of the American College of Surgeons 200.6 (2005): 965-972.
31. Tajima H., et al. "Hepatic arterial infusion chemotherapy with gemcitabine and 5 fluorouracil or oral S 1 improves the prognosis of patients with postoperative liver metastases from pancreatic cancer". Molecular and Clinical Oncology 1.5 (2013): 869-874.

32. Escobar MA., et al. “Solid pseudopapillary tumour (Frantz's tumour) of the pancreas in childhood: successful management of late liver metastases with sunitinib and chemoembolisation". Case Reports (2017): 221906.

\section{Assets from publication with us}

- Prompt Acknowledgement after receiving the article

- Thorough Double blinded peer review

- Rapid Publication

- Issue of Publication Certificate

- High visibility of your Published work

Website: www.actascientific.com

Submit Article: www.actascientific.com/submission.php

Email us: editor@actascientific.com

Contact us: +919182824667 